\title{
Mobility Management for multiple diverse applications in heterogeneous wireless networks
}

\author{
Robert Brännström ${ }^{1}$, Ruwini Kodikara E ${ }^{2}$, Christer Åhlund ${ }^{3}$, and Arkady Zaslavsky ${ }^{2}$ \\ ${ }^{1}$ Department of Computer Science, Luleå University of Technology, SE-971 87 Luleå, Sweden \\ ${ }^{2}$ School of Computer Science \& Software Engineering, Monash University, \\ 900 Dandenong Road, Caulfield East, Vic 3145, Melbourne, Australia \\ ${ }^{3}$ Division of Mobile Networking \& Computing, Luleå University of Technology, \\ SE-971 87 Luleå, Sweden \\ \{robert.brannstrom, christer.ahlund\}@1tu.se, \{piyangae, a.zaslavsky\}@csse.monash.edu.au
}

\begin{abstract}
This paper presents a mobility management solution to support both applications who are mobility-aware and those who are not. Mobility management in heterogeneous network environments needs to address the double meaning of the IP address as an endpoint identifier and a location identifier. Application-layer mobility use a non-IP endpoint identifier (e.g. user@ realm) while network-layer mobility uses a fixed home IPaddress as endpoint identifier. The resolving of the endpoint identifier to a temporary unicast IP address as location identifier needs support from a mobility management system. This paper proposes a mobility support system that integrates the benefits of application-layer SIP mobility with network-layer MIP mobility. A cross-layer information system provides context for mobility adaptation. Context awareness enhances handover decisions, transport performance and media adoption. The network-layer mobility supports the application to locate the destination ondemand for the initial setup of the sessions. The paper includes an initial evaluation of the network-layer mobility part of the solution.
\end{abstract}

Keywords-component; heterougeneous networks; mobility management; cross-layer exchange; seamless handover

\section{INTRODUCTION}

The deployment of wireless networks has made them ubiquitous and current research strives to make them pervasive. Users having wireless access to wired Internet Protocol (IP) networks and the Internet are driving the demand for mobile and heterogeneous solutions. Future wireless connectivity will be provided through a mix of coexisting heterogeneous access technologies. These access networks will adapt to the All-IP approach (4G) and contribute with different performance and coverage in a partially overlapping environment.

Current wireless networks like WLAN and GPRS/UMTS will be complemented by WiMAX and other evolving technologies. Multi-hop wireless ad hoc connections will be supported to enhance the service area of the access networks and provide forwarding of traffic into previous dead-spot areas. This requires a mobile host $(\mathrm{MH})$ to be equipped with multiple wireless network interfaces. Fig.1 shows and example of such heterogeneous network access that is already feasible today.

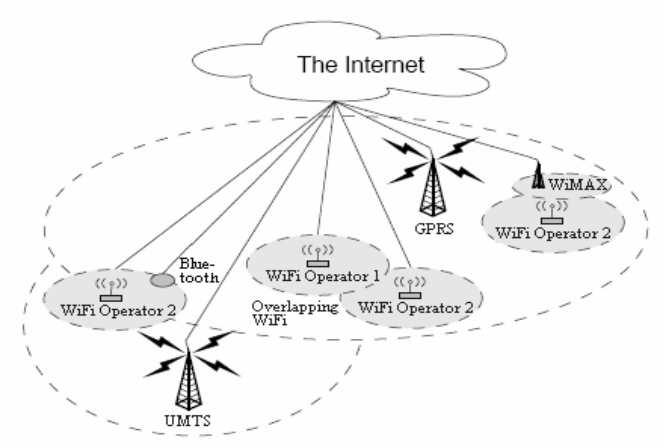

Figure 1. Wireless heterogeneous Internet access

Users could benefit from undemanding, self-configuring attachment to those ubiquitous networks in several ways. Through user mobility a user is enabled to switch between devices, migrate sessions and still get the same personalized services. Network mobility enables the users' devices to move around the networks and maintain connectivity and reachability. The general mobility problem can be regarded as an addressing and routing problem. More specific, the problem is the double meaning of the IP address as both an endpoint identifier and a location identifier [1]. The breakup of the double meaning could be handled at different layers in the network protocol stack and concerns different types of mobility. Using a non-IP personal address (e.g. user@ realm) as an endpoint identifier enables location transparent reach ability at the application level. This is the approach used by the Session Initiation Protocol (SIP) [2] who binds the unique identifier with a temporary unicast IP address. The combination of a permanent unicast IP address as endpoint identifier and a temporary unicast IP address as location identifier achieves location transparency at the network level and is the approach of Mobile IP (MIP) [3]. As indicated in Fig.2 the heterogeneous access have different effects on different types of mobility. By using a personal address the user could move herself or sessions between devices and by using a home address the devices could move between networks. 


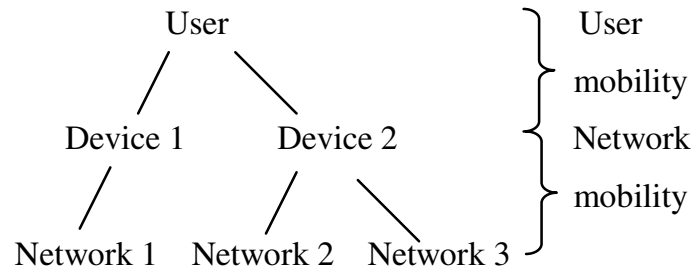

Figure 2. Mobility situation

Though MIP provides an elegant network-layer solution for seamless node mobility across IP networks its not the optimal mobility support solution for multimedia applications due to many reasons. Real-time multimedia applications are coupled with stringent delay requirements, are highly sensitive to packet losses and should be incorporated with a guaranteed response time. The indirect communication in MIPv4 increases the packet delay and the encapsulation overhead due to tunneling restricts the bandwidth utilization. Packet header overhead is particularly significant for low bit rate packet voice in which payloads is very short. On the other hand non realtime applications do not want to handle mobility and need the support from network-layer mobility.

We propose an integrated solution that blends the positive features of each protocol. The user identity (user@realm) is bound to the permanent home address of the user device and lets MIP handle the transportation to the current location. This solution enables the application to select the mobility method to use based on real-time or non real-time requirements.

Mobility management involves the decision of if, when and where to perform a handover to another network. Handover decisions could be triggered from coverage limitations, capacity demands or other specific requirements. The information needed for accurate and efficient handover could originate from any layer in the communication stack.

We propose a cross-layer information exchange system that enhances mobility adaptation (e.g. handover decision, adapting video quality etc).

The rest of the paper is structured in the following way. Section 2 describes a mobility scenario of a fully deployed system. Section 3 describes the architecture of the mobility support system and section 4 details the non real-time and the real-time mobility management. Section 5 describes related work and section 6 concludes the paper.

\section{MOBILITY SCENARIO}

To motivate the integrated mobility support system a scenario describes the benefits of a fully deployed system.

Sara, who is a research student, is on her way to the University by the morning train and participates in a videoconference with "media-IT", an industrial partner of the research project, using her laptop. She does not notice any disruptions although the train traverses several sub networks. (Real-time mobility situation)
After entering in to the University, Sara joins a seminar in a conference room. She exchanges electronic files with her colleagues through their laptops and downloads some web pages and files in-order to reinforce the seminar materials, working in the infrastructure mode. When she leaves the room and enters another room, the download process is continued. (Non real-time mobility situation)

Then she is supposed to join the e-meeting with her supervisors. She decides to continue the meeting from her office room and when entering the office she switches the session to her desktop PC. (Session mobility situation)

Later she arrives at another school situated in a different location, where she accesses a public desktop PC and receives an invitation from "media-IT" to join in another videoconference to discuss some urgent matters. "media-IT", unaware of Sara's mobility and current location, established the communication with Sara through her user id. To keep the confidentiality of the project Sara decides to switch the session to her mobile phone and moves outdoors to continue the meeting. (Personal mobility situation)

\section{MOBILITY SUPPORT ARCHITECTURE}

The description of the system in this paper refers to IPv4 solutions if not other stated. The mobility support system consists of Mobility Support Agents (MSA) at the home network and at the foreign network. The Home Mobility Support Agent (HMSA) provides the service of MIP home agent and SIP redirect/registrar server. The Foreign Mobility Support Agent (FMSA) provides the service of agent advertisement and IP address allocation. All functionality could be supported in a single server. To enable direct communication between end-hosts, the $\mathrm{MH}$ requires a colocated care-of address (CCoA) at the foreign network.

Important and related issues that are not included in the work at this stage are AAA, QoS, user profiles and policies and extended context awareness. We will address those questions in future work. Fig.3 shows an overview of the architecture and the pre-communication signaling.

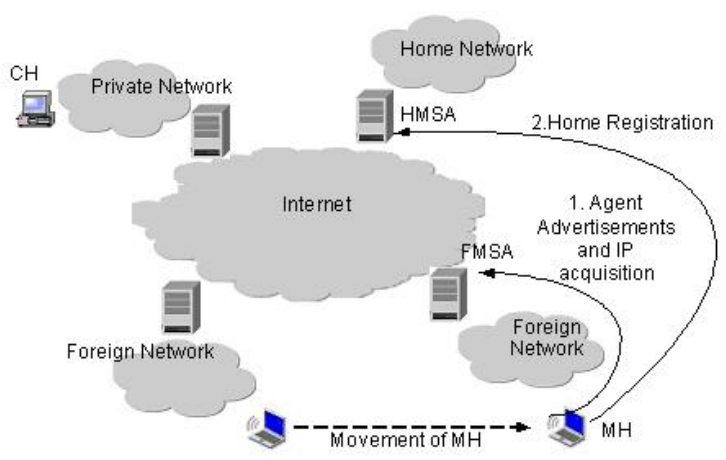

Figure 3. Mobility support architecture 


\section{MOBILITY MANAGEMENT}

The mobility management is supported at both the networklayer and at the application-layer. We choose to describe the mobility as network-layer and application-layer while the actual choice is taken by the application based on the real-time requirements. When the home address is used as IP source the network-layer will handle mobility by default and if the application chooses to handle mobility itself it must use the colocated care-of address as IP source.

\section{A. Network-layer mobility}

MIP is the standardized basic IP mobility support that provides seamless mobility without breaking TCP flows. Actual MIPv4 deployment would require reverse tunneling because of ingress filtering at the foreign network. The tunnel will have the HMSA and the CCoA as endpoints. M-MIP [4] is an extension to MIP that enables multihoming by accepting multiple bindings for a MH. This enables seamless handover without packet loss in a heterogeneous network environment. M-MIP uses RVM [5] and RNL [4] network-layer metrics in handover decisions.

Agent discovery (and mobility detection) is handled by MIP agent advertisements sent by the FMSA however the system also supports agent solicitation. The MH uses the advertisements to evaluate the network-layer capacity for handover decisions. When a $\mathrm{MH}$ detects a new network it acquires a CCoA from the FMSA. The MH then registers the new location with the HMSA and the M-MIP registration contains the preferred CCOA in case the device is multihomed. The HMSA stores the bindings for the $\mathrm{MH}$ and activates a tunnel to the selected CCoA. The binding is soft state and would timeout if not renewed. Fig. 4 shows the path of traffic handled by M-MIP and how the MH associates with several FMSAs.

In IPv6 the router advertisements and solicitation could be used and the IP address acquisition could by handled by stateless auto-configuration. This would remove the need of a MSA at the foreign network. The MIPv6 route optimization option would relive pressure from the HMSA by allowing direct communication between the end hosts (although encapsulated).

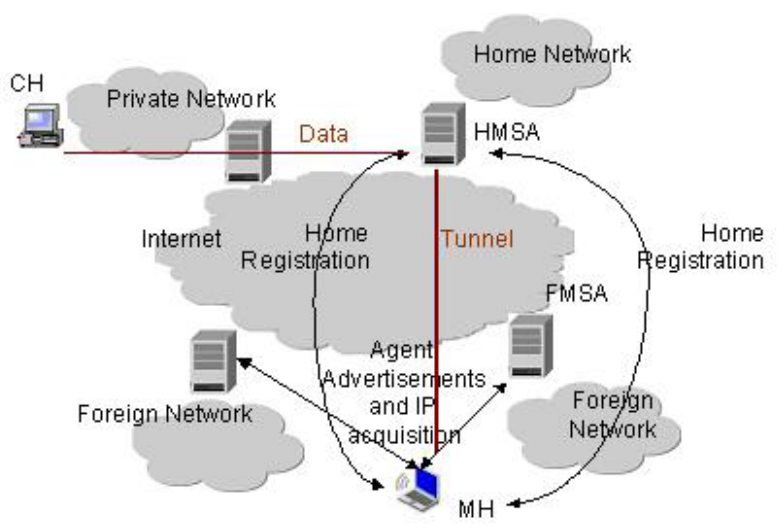

Figure 4. Multihomed network-layer mobility
An initial study of the network-layer mobility management implementation verifies the M-MIP ability of soft handover. In the study we use ping requests to an Internet peer $(\mathrm{CH})$ for handover evaluation. Packets are sent every 50 millisecond.

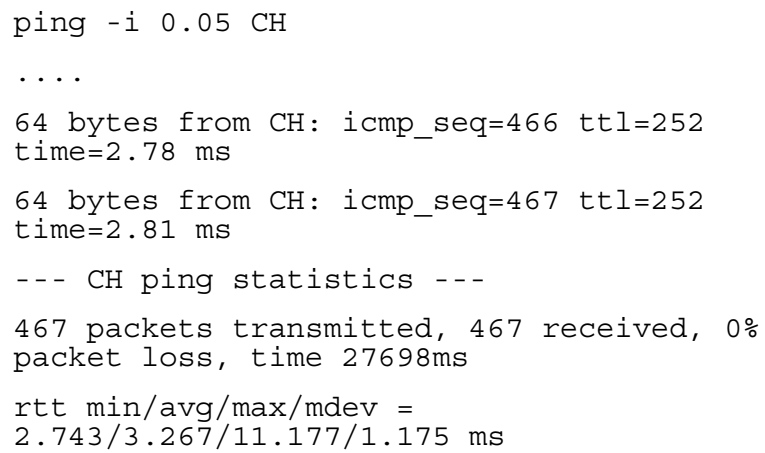

At the same time the $\mathrm{MH}$ moves from one foreign network through an overlapping area to another foreign network and performs the handover (see Fig. 4).

Connected to FMSA1

[i] FMSA1 has the best connection (RNL: new 0.007293 old 0.136873 )

[i] Current gateway is FMSA1

Connected to FMSA2

[i] FMSA2 has the best connection (RNL: new 0.006053 old 0.007566 )

[i] Current gateway is FMSA2

This test verifies that no packets were lost during the soft handover.

\section{B. Application-layer mobility}

SIP is an application-layer protocol that handles establishment of real-time sessions as well as session migration. These features could be used to achieve personal mobility and session mobility as well as device mobility. The mobility support system enables personal mobility by SIP registering of user device. The user registers the home address (HoA) of the current device at the HMSA which creates the binding to the user identity. Fig. 5 shows how the non real-time initial invitation is routed through the home network while the following signaling and the real-time traffic are routed directly between the end hosts.

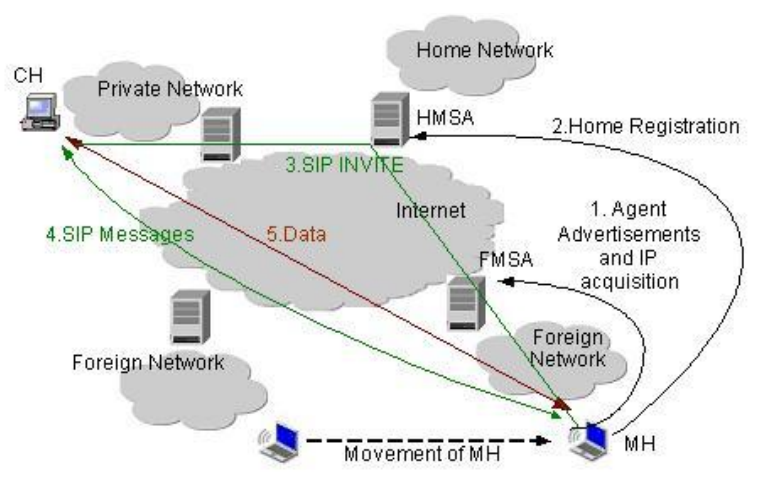

Figure 5. Application-layer mobility 


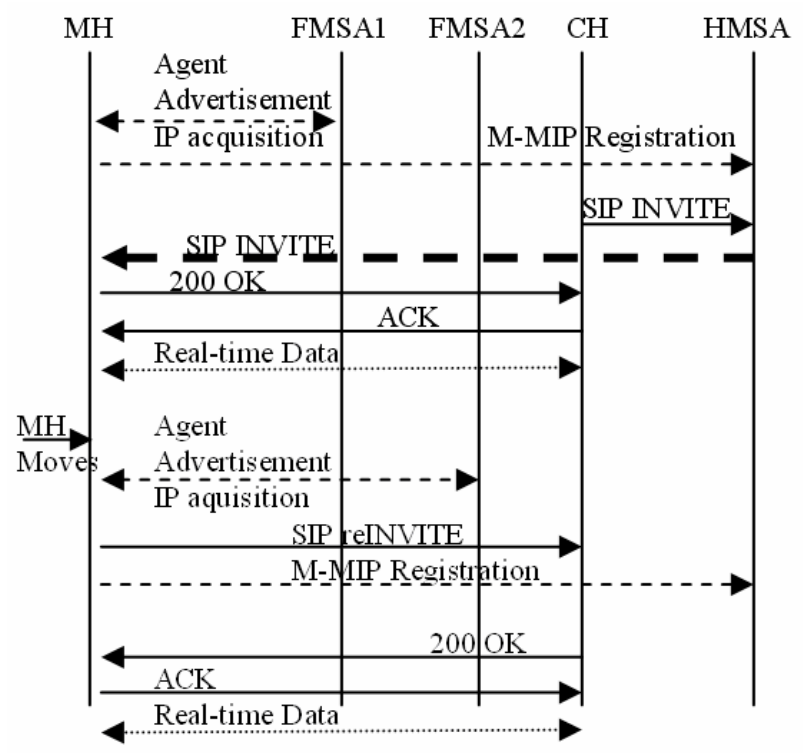

Figure 6. Message sequence for mid-session mobility

Whenever an invitation arrives at the HMSA the user identity is resolved to a device home address and the invitation is forwarded to the current location through M-MIP tunneling. M-MIP maintains multiple registrations and selects the best one to use. At reception of the invitation the $\mathrm{MH}$ replies directly to the $\mathrm{CH}$, establishing a direct path used by further SIP signaling and real-time data as shown in Fig. 6.

Session mobility is accomplished by the SIP reINVITE message. The reINVITE message includes the new location and an updated session description. The SIP messages are exchanged directly between the $\mathrm{MH}$ and the $\mathrm{CH}$ and thereby minimize the handover delay. After the handover is done the $\mathrm{MH}$ register the new location with the HMSA according to the network-layer description. The mid-session message sequence is shown in Fig. 6.

The approach is similar when the user decides to move the session to another device. A user may be associated with multiple devices at the same time and could transfer one or all session to another device by a reINVITE message. A user could also decide to use multiple simultaneous connections to support redundant streams in areas with interference or when performing handover.

As well as the user may be in possession of several devices she may have several user identities or alias (e.g. a professional identity and a private identity). These user identities might be associated with different or the same email addresses, phone numbers etc. Associations are maintained by SIP registrations with the HMSA. When the HMSA receives an INVITE it checks for user preferences matching the user identity to an active device.

The mobility support solution separates the user from the device and the current location, enabling true mobility that supports all kind of applications.

\section{CROSS-LAYER INFORMATION}

To support the real-time applications in mobility management and data adoption a cross-layer information exchange system provides with available context. The context is available at all layers and provides information to be used by all layers as they wish.

The cross-layer information module is at this stage focused to mobility related context exchange and adoption. We do not for example include issues related to QoS adaptation and Channel adaptation of multimedia sessions.

Mobility related context can be exchanged between layers to make the mobility adaptation more efficient. Link-layer parameters like signal strength, handoff initiation and completion events can be used to make network-layer handover decisions more efficient. Link-layer parameters like packet loss and bit-error-rate could also be used to fine tune the performance at the transport-layer (e.g. retransmission and back-off) and for adoption at the application-layer (e.g. media encoding and transmission rate).

The network-layer notifications and mobility information can be used to enhance the application-layer movement detection (e.g. new IP address). Even if the network interface automatically receives a new IP address the application is not informed and it has to perform repeated polling of the interface to detect mobility.

Cross-layer information exchange support service differentiation based on higher layer requirements. If a device has multiple wireless network interfaces the selection of the appropriate interface to use could be based on user needs or multimedia application requirements. An example is when an application is coupled with stringent delay requirements the WLAN interface can be used for a better throughput. But if lower delay requirements are tolerable, the device can switch to the GPRS interface.

Fig.7 shows an overview of the cross-layer information exchange flow.

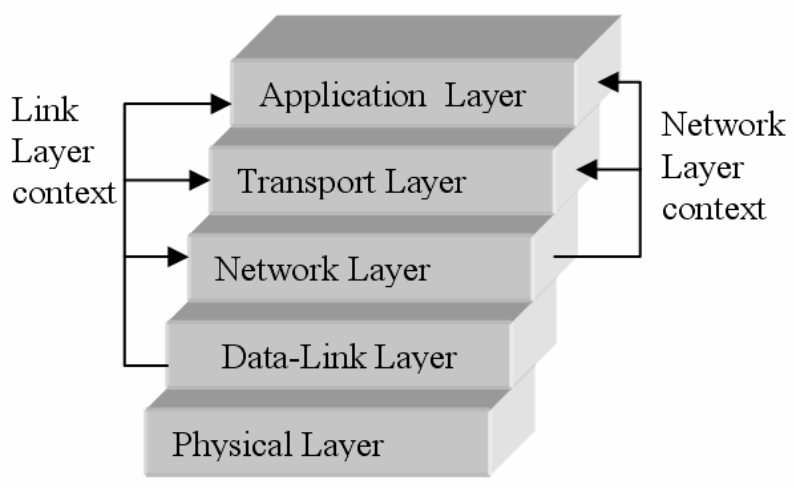

Figure 7. Cross-layer information exchange 


\section{RELATED WORK}

Application-Layer Mobility Using SIP [6] describes how SIP can provide terminal, personal, session and service mobility. It also describes that MIP should be preferred for terminal mobility.

Mobility Support using SIP [7] proposes mobility support at the application-layer for real-time communication. They propose to use the HA for location lookup or to let SIP redirect the invitation to the home address of the $\mathrm{MH}$ and let the HA forward id to the $\mathrm{MH}$.

Multilayer Mobility Management for All-IP Networks: Pure SIP vs. Hybrid SIP/Mobile IP [8] evaluates the protocols ability to handle real-time sessions. Through evaluation they show the SIP superiority in real-time situations while MIP is preferred in non real-time situations. The paper investigates possibilities of combining the two protocols into a hybrid solution.

Integrated Mobile IP and SIP approach for advanced location management [9] expresses the benefits of an integrated solution to a hybrid solution to avoid redundant signaling. A Mobility Server is proposed to handle all mobility related functionality (e.g. all SIP server functionality, IP address distribution, AAA and forwarding agent).

The Mobile people architecture [10] introduces the concept of personal routing between people that could be hosted on different devices. They focus on personal adoption and media conversion to handle cell phones that are turned off, or PCs on faraway desktops.

A Multi-Layer Mobility Management Architecture Using Cross-Layer Signaling Interactions [11] express the benefits of cross-layer information for enhancement in mobility management. It also identifies each layer's contribution in various mobility support tasks.

Mode switching and QoS issues in software radio [12] describes how a mobile device can be reconfigured to satisfy application requirements. The authors propose a switching between different air-interface standards while according to QoS requirements.

Simple but effective cross-layer networking system for mobile ad hoc networks [13] explains how adaptive routing can be achieved based on low-layer information.

Wireless Channel-Aware Ad Hoc Cross-Layer Protocol with Multi-Route Path Selection Diversity [14] proposes how route discovery and route optimization could be based on channel information.

\section{CONCLUSIONS}

This paper proposes solutions towards enabling and supporting all types of mobility in heterogeneous networks. The mobility support system provides mobility management at both network-layer and application-layer through Mobility Support Agents.

Our solution supports non real-time applications by the network-layer mobility functionality. This keeps the application unaware of network mobility and works as a backup for real-time applications.

Application-layer mobility supports personal and session mobility for mobility aware real-time applications. The integrated solution uses the network-layer forwarding of initial INVITE messages.

Cross-layer information exchange contributes to mobility adoption at all layers of the protocol stack. Context awareness enhances handover decisions, transport performance and media adoption.

In future work we will implement full mobility support and evaluate the solution in heterogeneous mobility scenarios.

\section{REFERENCES}

[1] A. C. Snoeren, H. Balakrishnan, M. F. Kaashoek. "Reconsidering Internet Mobility," Proc. 8th Workshop on Hot Topics in Operating Systems HotOS VIII, May 2001.

[2] J. Rosenberg, H. Schulzrinne, G. Camarillo, A. Johnston, J. Peterson, R. Sparks, M. Handley, and E. Schooler. SIP: Session Initiation Protocol (IETF RFC 3261). 2002.

[3] C. Perkins. IP Mobility Support for IPv4, Internet Draft. 2004.

[4] C. Ahlund, R. Brannstrom, and A. Zaslavsky, "M-MIP: extended Mobile IP to maintain multiple connections to overlapping wireless access networks," International Conference on Networking, Apr. 2005.

[5] C. Ahlund, R. Brannstrom, and A. Zaslavsky, "Running Variance Metric for evaluating performance of Wireless IP Networks in the MobileCity Testbed," International Conference on Testbeds and Research Infrastructures for the Delevopment of Networks and Communities, Feb. 2005.

[6] H. Schulzrinne and E. Wedlund, Application-layer mobility using SIP SIGMOBILE Mob. Comput. Commun. Rev., vol. 4, pp. 47--572000.

[7] E. Wedlund and H. Schulzrinne, "Mobility support using SIP," Proceedings of the 2nd ACM international workshop on Wireless mobile multimedia, 1999.

[8] C.Politis, K. Chew , and R. Tafazolli, "Multilayer Mobility Management for All-IP Networks: Pure SIP vs. Hybrid SIP/Mobile IP," 57th IEEE Semiannual Vechicular Technology Conference VTC2003, Apr. 2003.

[9] Q. Wang and M. A. Abu-Rgheff, "Integrated Mobile IP and SIP Approach for Advanced Location Management," Proc. IEE 4th International Conference on 3G Mobile Communication Technologies 3G2003, June 2003.

[10] Mobile People Architecture. 99.

[11] Q. Wang and M. A. Abu-Rgheff, "A Multi-Layer Mobility Management Architecture Using Cross-Layer Signalling Interactions," Proc. IEE 5th European Personal Mobile Communications Conference EPMCC'03, Apr. 2003.

[12] A. Aghvami, T. Le, N. Olaziregi, "Mode switching and QoS issues in software radio," IEEE Personal Communications 8 (5) (2001) 38-44.

[13] Wingho Yuen, Heung-No Lee and Timothy Anderson, "Simple but effective cross-layer networking system for mobile ad hoc networks," Proc. of IEEE PIMRC, pp. 1952-6, Lisbon, Portugal , Sept. 2002

[14] M. Park, J. G. Andrews, and S. Nettles, "Wireless Channel-Aware Ad Hoc Cross-Layer Protocol with Multi-Route Path Selection Diversity", IEEE Vehicular Technology Conference, Orlando, FL, October 2003. 